\title{
Gestión de los Colegios Profesionales de Educación Física y Deporte desde una perspectiva interna \\ Management of Professional Colleges of Physical Education and Sport from an internal perspective

\author{
*Roberto Silva Piñeiro, **Jorge Fernando García Unanue \\ *Universidad de Vigo (España), **Universidad de Castilla-La Mancha (España)
}

\begin{abstract}
Resumen. Asistimos en la última década a un intenso crecimiento del ámbito deportivo y en consecuencia también a la aparición de nuevas profesiones. Los Colegios Profesionales de Educación Física y Deporte garantizan la calidad y seguridad de los servicios cualificados, pero también se han visto afectados por diversas normativas y obligaciones que han lastrado su capacidad operativa. Se propuso un estudio de funcionamiento de estas entidades a nivel estatal que ayudase a interpretar el presente y el futuro más inmediato que tendrán que afrontar. Objetivo. Describir en qué situación se encuentra una parte de la gestión interna de los COLEF. Metodología. Basándonos en diferentes normativas y manuales, se diseñó un formulario online sobre cuestiones organizativas que fue trasladado a cada colegio autonómico. Resultados. Se obtuvo una participación de 15 entidades, recogiéndose datos que muestran algunos nexos estructurales, carencias, posicionamientos y tendencias organizativas similares. Conclusiones. Se detectó una ausencia en la utilización de procesos y herramientas de planificación. Asimismo, los COLEF necesitan profundizar en el conocimiento de su cliente interno e incrementar servicios y estrategias de fidelización, además de mejorar en general todas sus relaciones. A pesar de que la austeridad económica y dependencia de las cuotas anuales, no se detectaron tensiones de liquidez ni deuda financiera, lo que también favorecería el planteamiento de nuevos retos estructurales.
\end{abstract}

Palabras clave. Colegio Profesional, Colegiados, Comunicación interna, Educación Física y Deporte, Gestión.

\begin{abstract}
In the last decade, we have witnessed an intense growth of the sport field and consequently also the emergence of new professions. The Professional Collegues of Physical Education and Sport (COLEF) guarantee the quality and safety of qualified services, but they have also been affected by various regulations and obligations that have hindered their operational capacity. A study of the functioning of these entities at state level was proposed to help interpret the present and the more immediate future they will have to face. Objectives. To describe the current situationt of a part of the internal management of the COLEF. Methodology. Based on different regulations and manuals, an online form was designed on organisational issues and sent to each regional college. Results. The participation of 15 entities was obtained, collecting data that show some similar structural links, shortcomings, positionings and organisational trends. Conclusions. An absence in the use of planning processes and tools was detected. Furthermore, COLEFs need to deepen their knowledge of their internal customers and increase services and loyalty strategies, as well as generally improve all their relationships. Despite the economic austerity and dependence on annual fees, no liquidity tensions or financial debt were detected, which also would favours the approach of new structural challenges.
\end{abstract}

Keywords. Professional Collegue, Collegiates, Internal Comunication, Physical Education and Sport, Management.

\section{Introducción}

Estamos asistiendo con intensidad en esta última década a la aparición de nuevas profesiones, reivindicativas, necesarias y con un impacto socio-económico. Algunas de ellas están en el ámbito de la actividad física, la educación física y el deporte, por lo que, las entidades que las representan, particularmente los Colegios Oficiales o Profesionales de la Educación Física y Deporte, como se puede consultar en sus Estatutos (BOC, 2009;

Fecha recepción: 06-09-21. Fecha de aceptación: 24-01-22 Roberto Silva Piñeiro

roberto.silva@uvigo.es
BOCL, 2018; BOCM, 2014; BOCV, 2000; BOLR, 2018; BON, 2014; BOPA, 2017; BOIB, 2014; BOJA, 2018; BOPV, 1998, BORM, 2002; DOCM, 2013; DOE, 2005; DOGC, 2000), se muestran como una referencia para muchos sectores, también emergentes. El reconocimiento social que puedan alcanzar y su capacidad de organización afectan a la actividad profesional y servicios de miles de trabajadores, de ahí el inherente interés que tiene su estudio, considerando la escasa investigación al respecto.

El colectivo profesional de Educadores/as Físico Deportivos/as se caracteriza por su variedad y dinamismo, así como por su gran capacidad de adaptación y crecimiento. Sin embargo, también muestra las debili- 
dades afectadas por la velocidad a que se están produciendo los acontecimientos a nivel social, sectorial y colegial, que conlleva un déficit reflexivo y una mirada oblicua a futuros y complejos escenarios sin las adecuadas herramientas.

La Ley 2/1974, de 13 de febrero, sobre Colegios Profesionales, estableció que los colegios son Corporaciones de derecho público, amparadas por la Ley y reconocidas por el Estado. Entre sus fines está la ordenación del ejercicio de las profesiones, la representación institucional exclusiva de las mismas cuando estén sujetas a colegiación obligatoria, la defensa de los intereses profesionales de los colegiados y la protección de los intereses de los consumidores y usuarios de los servicios de sus colegiados. Entre sus funciones encontramos la protección de consumidores y usuarios ante los servicios de sus colegiados; realización de estudios, informes y otras actividades para las administraciones; participación en los consejos u organismos consultivos de la administración en la materia de competencia de cada una de las profesiones; mantenimiento del contacto y preparación de la información necesaria para facilitar el acceso a la vida profesional de los egresados; ostentación en su ámbito la representación y defensa de la profesión; facilitación de un listado de peritos colegiados para asuntos judiciales; control dentro de su competencia, por la ética y dignidad profesional de los colegiados; organización de actividades y servicios comunes de interés para los colegiados; y adopción de las medidas para evitar el intrusismo profesional.

También conocemos que los Colegios Profesionales pueden realizar la función de mediación (Unión Profesional, 2013), un método que facilita la resolución de conflictos sin tener que acudir a la justicia o el arbitrio. En esta situación la presencia de un mediador es fundamental para conseguir un acuerdo entre partes, razón por la que la modificación de la Ley 2/1974 de Colegios Profesionales los considera instituciones de mediación, función que ya realizaban desde hace años, pero para la que es necesario una formación específica.

Por su parte, relacionado con la organización de actividades de carácter formativo para los profesionales colegiados, la misma normativa contempla que puedan tener representación en los Patronatos Universitarios, participar en la elaboración de los planes de estudio, organizar cursos para la formación profesional de los postgraduados. No obstante, el desarrollo de estas funciones depende mucho de las relaciones de cada Colegio Profesional con las respectivas Universidades. En España, únicamente en dos ámbitos, la Administración cuenta con un sistema de certificación de la formación continua de los profesionales, el educativo y el sanitario, pues es la principal empleadora. Aunque en ocasiones ha sido cuestión de debate, un sistema de formación continua global, y en particular que atendiese al ejercicio profesional privado, sería muy costoso. En este sentido, son los Colegios Profesionales los que conocen mejor las particularidades de su profesión, y pueden certificar que los servicios a los que se les concede son objeto de las evaluaciones y controles que respondan a normas o protocolos que deberán cumplir los colegiados en la prestación de los servicios. Esto conllevaría establecer servicios colegiales de certificación de calidad e inspección del servicio profesional, diferenciando a aquellos que prestan sus servicios con la calidad garantizada por el colegio de aquellos que no se hayan sometido a tal evaluación (Chacón, 2009).

Dado que es una cuestión principal, en la que la normativa comunitaria está fortaleciendo la integración de la formación formal con la no formal e informal, es necesario atender a cómo se pueden ir organizando las carreras profesionales en los nuevos escenarios competitivos. Según Unión Profesional (Múzquiz, 2013), el Desarrollo Profesional Continuo (DPC) es el medio por el que una persona mejora constantemente sus conocimientos y técnicas en un área profesional determinada va adquiriendo una creciente importancia en el mundo profesional moderno, especialmente debido a la necesidad de los nuevos profesionales de ir adaptándose a unas necesidades cada vez más cambiantes que requieren diversas cualificaciones, capacitaciones y habilidades. Por ello los Colegios Profesionales tienen que estar considerados para su acreditación y certificación, basándose en los principales modelos de DPC europeos.

Relacionado con la visibilidad profesional y estatus social, Palomar (2019), insiste en que la vinculación con la salud, la seguridad, la protección de colectivos específicos, de los consumidores y el medio ambiente, también justifican la regulación de la profesión para aquellos que finalicen el Grado en Ciencias de la Actividad Física y del Deporte. En este punto, Díez y Gambau (2019), consideran que es necesario resolver la denominación profesional para regular de forma conveniente y estar en la línea del test de proporcionalidad que requiere la Unión Europea para las regulaciones profesionales (Directiva 2018/958 del Parlamento Europeo y del Consejo, de 28 de junio de 2018). En el año 2013 se acordaba tramitar la denominación de Colegios Profesionales de la Educación Física y del Deporte para los colegios autonómicos, coincidiendo con la extinción del 
título de Licenciado, y establecer el nombre de los profesionales como Educadores/as Físico Deportivos/as.

Según Gambau (2018), esta profesión se inicia con el Colegio Nacional de Profesores de Educación Física de España (1948-1978), para a continuación transformarse en profesión colegiada tras la Ley de Colegios Profesionales de 1974. En 1978 se creó el Colegio Oficial de Profesores de Educación Física y por lo tanto la construcción jurídica de la profesión de Profesor/a de Educación Física en España. En 1981 se produjo la constitución por segregación de los Colegios Oficiales de Profesores de Educación Física de Cataluña y el País Vasco, y constitución del Consejo General de Colegios de Profesores de Educación Física de España y de más Colegios Oficiales autonómicos, que duraría hasta 2003. Con la creación de la Licenciatura de Ciencias de la Actividad Física y del Deporte en los años 1990, los Colegios autonómicos fueron modificando su denominación para acoger tanto esta titulación universitaria como su conversión al grado. Por tanto, la profesión se fue adaptando a la evolución de las titulaciones universitarias.

Como era previsible, la situación de pandemia ralentizó durante 2020 y 2021 el crecimiento de los colegios autonómicos de educadores físicos y deportivos de España, fundamentalmente en aquellos con mayor tamaño (Consejo COLEF, 2021). Pese a todo, el colectivo ha seguido aumentando, llegando a los 16.338 profesionales. Se trata de un grupo joven, pero no reciente, por lo que a partir de ahora comenzaremos a observar el comportamiento vegetativo de colegiación que no se había experimentado. Acorde con la menor tasa de matriculación femenina en los estudios universitarios, esta crisis ha venido a certificar también, el descenso que desde el 2016 se viene produciendo en el número de mujeres colegiadas, que no alcanza el 30\% de participación.

En cuanto a la distribución territorial de la colegiación, se destacan cuatro comunidades autónomas (Cataluña, Madrid, ComunidadValenciana y Andalucía), aunque per cápita también tendrían relevancia Galicia, Islas Baleares e Islas Canarias. Con respecto a otras profesiones (Figura 1), la de Educación Física y Deporte constituye un porcentaje reducido, al menos con respecto a las profesiones sanitarias, arquitectura, ingenieros y abogados. Sin embargo, es importante tener en cuenta las tendencias que se arrastran, que en el caso de las profesiones sanitarias y también en la Educación Física y Deportiva, al contrario de las técnicas y jurídicas, es ascendente en los últimos años.

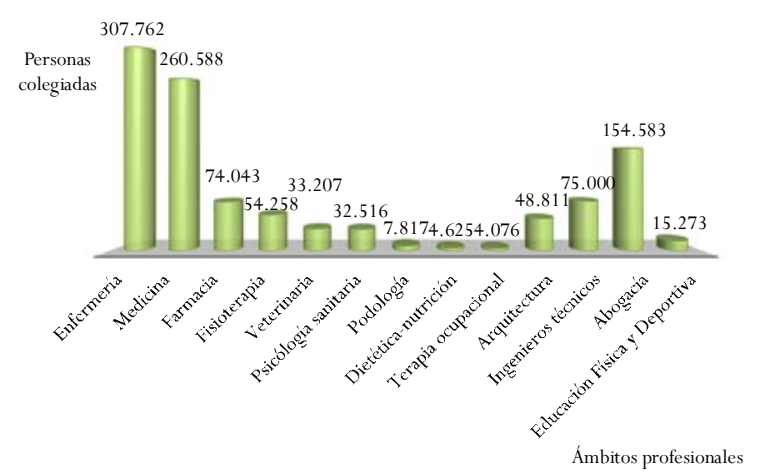

Figura 1. Colegiación por profesiones en España en 2018. Fuente: INE (2019), CSCAE (2019), Consejo COLEF (2019).

Hace poco más de veinte años, aunque algunos fuesen poco visibles y utilizados, se consideraba que el núcleo de un Colegio Profesional eran los servicios colegiales, servicios jurídicos, publicaciones, formación y servicios telemáticos. Unos facilitan la toma de decisiones en cuestiones de impacto profesional, y otros localizan los problemas con los que se puede encontrar repetidamente la persona colegiada, e intenta minimizarlos. El valor añadido de estos servicios no es su frecuencia de uso, sino la tranquilidad de saber que podemos contar con ellos si los necesitamos (Elías \& Mascaraya, 1998).

Para Chacón (2009), en países como Italia o Portugal los Colegios Profesionales tienen una naturaleza jurídica similar a la nuestra, pero en los anglosajones es distinta, donde la pertenencia aparentemente no es obligatoria pero la Administración le delega el reconocimiento de la cualificación profesional, y por tanto la capacidad de determinar los requisitos y puedan expedir títulos formativos con derecho a utilizarlos profesionalmente. Esto es una ventaja con respecto a nuestro modelo de Colegios Profesionales, pues estas asociaciones, además del control profesional pueden definir el tipo de formación que da acceso a la profesión.

La Ley de Transparencia 19/2013, refuerza que los Colegios Profesionales cuenten con una estructura interna y un funcionamiento democrático, y posibiliten la intervención de los profesionales en la vida pública dando oportunidad a inquietudes de manera organizada. Entre las opciones de participación están, por un lado, la implicación del profesional en las iniciativas de colegio a través de formación e información y, por otro, la implicación de colegio en las iniciativas de los profesionales, ofreciendo los medios, herramientas y canales adecuados para ello, generando un circuito abierto que posibilita que profesionales, institución y sociedad se vayan nutriendo y alimentando de iniciativas de origen profesional e impacto social. 
Uno de los inconvenientes a la hora de maximizar los beneficios es la evaluación exacta de la aportación de las entidades colegiales en su ámbito de actividad, por eso se incorporan procedimientos más precisos para medir el progreso y consecución de los fines sociales y profesionales, con el objetivo de demostrar que la contribución social es mayor que el coste de ejecución (Sánchez, 2015). A este respecto, Unión Profesional (2016) publicó una guía para la implantación en el sector colegial del Buen Gobierno con tres niveles diferenciados. El primero centrado en la gobernanza interna en los Colegios Profesionales respecto a sus colegiados, el segundo sobre las relaciones de Buen Gobierno de los Consejos Generales con sus respectivos colegios y delegaciones, y un tercero orientado hacia una gobernanza global entre las distintas profesiones, orientada a la búsqueda de soluciones conciliadoras.

Este estudio se propuso describir en qué situación se encuentra una parte de la gestión de los Colegios Profesionales de Educación Física y Deporte en España (COLEF). Sin mayor pretensión de profundizar y partiendo de la base de que gestión y gerencia se presentan como sinónimos en el idioma castellano y en numerosas ocasiones se utilizan al referirse a un evento administrativo en general, existe una tendencia a diferenciarlos en base a su eficacia. El término gestión, en cuanto a los colegios profesionales, se utiliza para designar a la administración que busca los fines corporativos, entendiéndose fuertemente los vinculados a sus políticas y organización general. Por su parte, gerencia se emplea frecuentemente para referirse a la búsqueda de la optimización y búsqueda de eficiencia, incluyendo la económica (Contreras \& Crespo, 2005). El trabajo podría ayudar a mejorar las decisiones directivas y de gerencia, verificar el cumplimiento de las finalidades corporativas, analizar las fortalezas y debilidades de las corporaciones regionales, así como a exponer los aspectos a corregir y potenciar en los COLEF autonómicos para fortalecer su eficiencia organizativa.

\section{Material y método}

Se trata de un estudio transversal y descriptivo, con un enfoque cuantitativo-cualitativo, ya que era pertinente conocer también las opiniones, experiencias y demás aspectos subjetivos de los participantes (Gibbs, 2012; Hernández, Fernández \& Baptista, 2014), optándose por un diseño de análisis a detalle de unidad de investigación grupal (Merriam, 1998). Se optó por un marco de referencia deductivo para la conceptuali- zación y generación de categorías (Osses, Sánchez \& Ibáñez, 2006). De la revisión de la literatura se obtuvieron las codificaciones para identificar los componentes temáticos de referencia, Con la supervisión de dos expertos independientes.

\section{Instrumentos}

Se utilizó un formulario ad hoc, teniendo en cuenta lo emanado del Reglamento de Régimen Interno y Código Deontológico, se apoyó en las directrices y recomendaciones que se recogen en el Manual de Buen Gobierno en asociaciones del Consejo Superior de Deportes (2004), la Guía de Profesiones Colegiadas de Unión Profesional (2020), los Estatutos publicados de los diferentes COLEF autonómicos (1998-2020), el Código de Buen Gobierno de la Organización Colegial de la Educación Física y Deportiva (2019), que sería cumplimentado por un responsable (profesional o directivo) de cada Colegio Profesional. Esta herramienta estaba formada de 22 preguntas temáticas sobre relaciones institucionales, estructura y organización, servicios y comunicación, con opciones variables: 1 de respuesta abierta, 5 de respuesta mixtas y 16 de opción cerrada. Aleatoriamente, una vez confeccionado el cuestionario, se eligieron dos COLEF para realizar los ajustes necesarios y comprobar si alcanzaba para analizar los diferentes apartados propuestos.

\section{Muestra}

Participaron 15 Colegios Profesionales de Educación Física y Deporte en España, de un universo muestral de 17 entidades.

\section{Resultados}

La participación activa de los colegios autonómicos en la organización universitaria no alcanza el 33\%, destacando que el $53 \%$ admite tener funciones en comités de deporte autonómico, y el $26 \%$ no las tienen en universidades, deporte autonómico ni plataforma sectoriales (Figura 2).

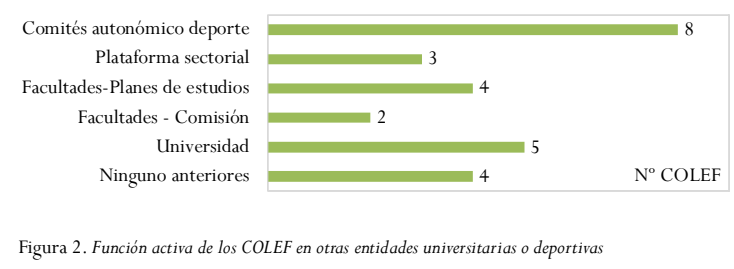

Todos los COLEF analizados tienen contacto con los respectivos departamentos autonómicos de deportes, 
seguidos de las facultades y departamentos autonómicos de educación. Por otra parte, tan sólo un 6\% mantiene relación activa con sindicatos, partidos políticos, federaciones o departamento autonómico de trabajo; destacando que ningún colegio mantiene relación con alguna oficina o asociación de consumidores. En el caso de relaciones con colegios profesionales de otros ámbitos, destacan fundamentalmente los de la rama sanitaria, en particular dietética-nutrición y medicina; aunque también un 33\% de los colegios no mantiene ninguna relación actualmente (Figura 3).

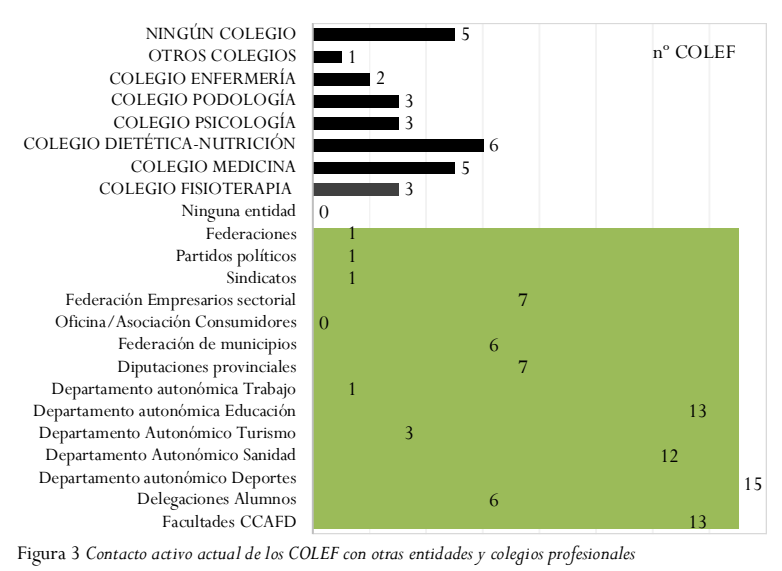

El 66\% de los COLEF reconoce que realiza asesoramiento a entidades y peritajes técnicos, y el $46 \%$ ofrece certificados/sellos de calidad. Ninguno ofrece conciliación/mediación, y un $13 \%$ no cuenta con ninguno de los servicios consultados. La mayor parte de los COLEF tiene derivadas las asesorías (jurídica, laboral y fiscal), mientras que el 40\% tiene contratada los servicios de contabilidad y comunicación, el 13\% los servicios informáticos, y un colegio no tienen ningún contrato externalizado (Figura 4).

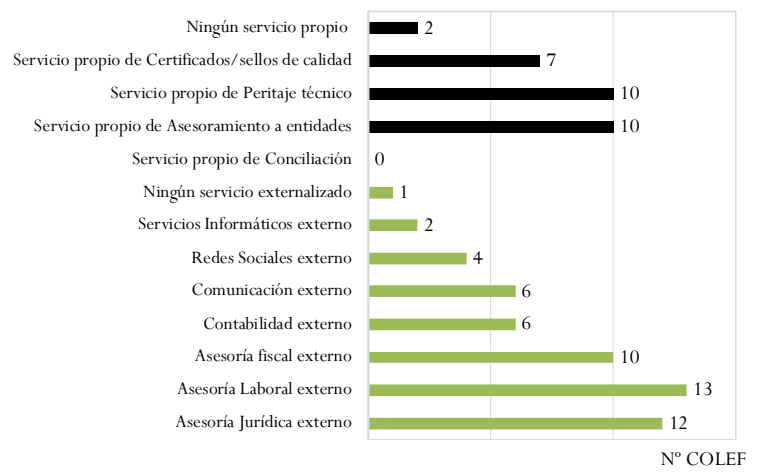

Figura 4 Prestación y externalización de servicios de los COLEF

Los COLEFs destaca que el 23\% de los colegios autonómicos cuentan con manual de imagen corporativa y firma electrónica, el 37\% de los colegios autonómicos admite contar con un programa de prevención global de riesgos, y el 16\% con un plan de comunicación. Sin embargo, un 19\% no tiene ninguna de las opciones digitales, participativa o de comunicación implementada, y ninguno cuenta con un plan de desarrollo digital ni de profesionalización de sus trabajadores (Figura 5).

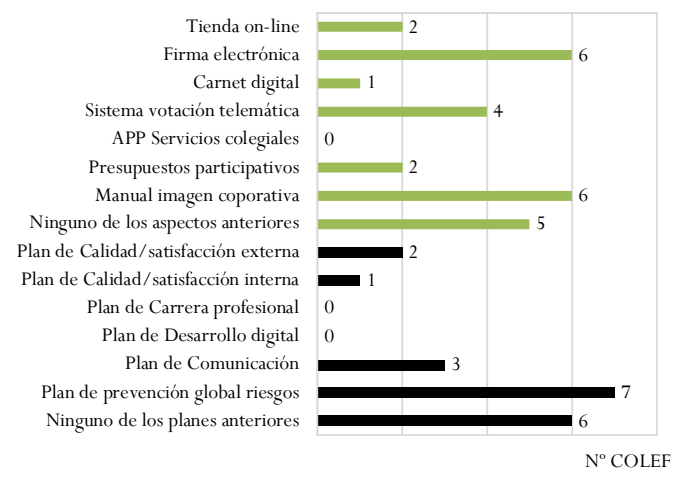

Figura 5 Implementación organizativa y planes específicos de los COLEF

Los COLEFs realizan una media de 2,13 formaciones anuales, teniendo en cuenta que los que más organizan son Andalucía (8,3/año), Cataluña (5,3/año), y Asturias (5/año). Por su parte tanto País Vasco como Navarra, Murcia e Islas Baleares no reconocen ninguna. Salvo los colegios catalán y aragonés, la mayor parte organiza entre 1-3 jornadas divulgativas anuales (Figura $6)$.

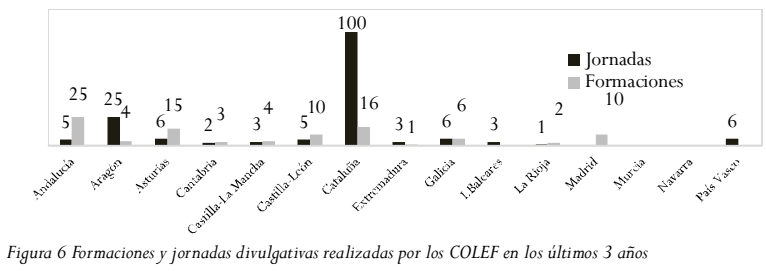

El equipo profesional de los COLEF lo forman una media de 2,33 personas, destacando el colegio catalán, con 13. Salvo el colegio cántabro y el riojano, que no cuentan con equipo profesional, la mayor parte los conforman entre 1-2 personas. El 87\% de los colegios tienen equipo profesional. El 66\% no cuenta con trabajadores a tiempo completo, y entre los que los tienen, la mayoría no es más de un trabajador/a. El 80\% tiene personal contratado a tiempo parcial, la mayoría entre 1-2 trabajadores, salvo Cantabria, Castilla-León y La Rioja que no cuentan con ninguno. Sólo el 13,3\% tiene personas becarias en su equipo profesional, entre 1-2. Los equipos profesionales tienen al menos un trabajador/a con la Titulación Universitaria en Ciencias de la Actividad Física y del Deporte, que conforman más de la mitad de las plantillas profesionales (Figura 7). 


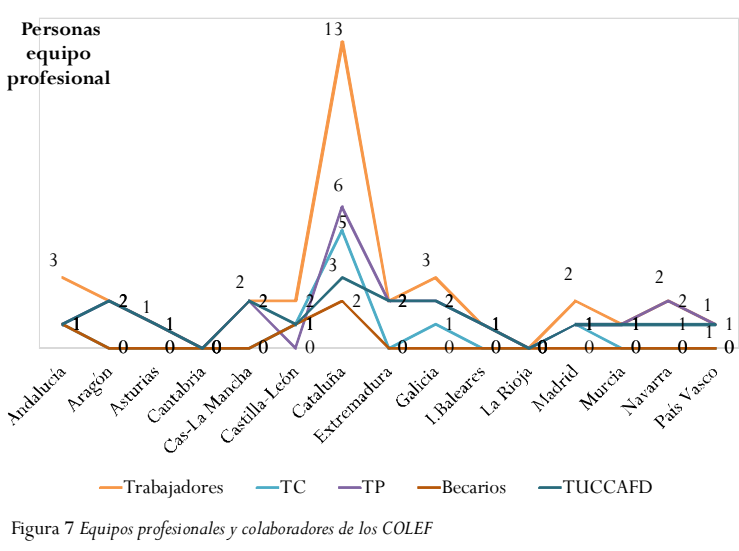

El porcentaje presupuestario al que contribuyen las cuotas colegiales es ligeramente superior al 90\%. Sólo el $27 \%$ de los colegios reconoce que alguna ocasión ha sufrido estrés de tesorería regular en los últimos tres años, y el 93\% de los colegios autonómicos no tienen contraída deuda bancaria o crédito privado (Figura 8).

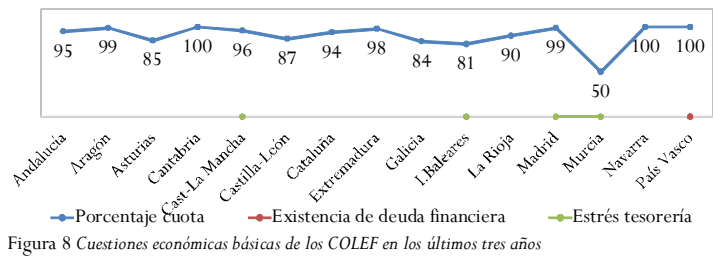

Los COLEF consultados consideran prioritario esforzarse en potenciar la comunicación, reforzar el equipo profesional y mejorar los servicios a la colegiación, y la formación. Apenas están interesados en potenciar la organización interna, las relaciones institucionales o los servicios a terceros. Por su parte consideran estratégico para el futuro mejorar las alianzas, las reclamaciones judiciales y los avances normativos; alejándose de esfuerzos para planificar la captación de colegiados, los proyectos sociales o el diálogo interno de la propia entidad (Figura 9).

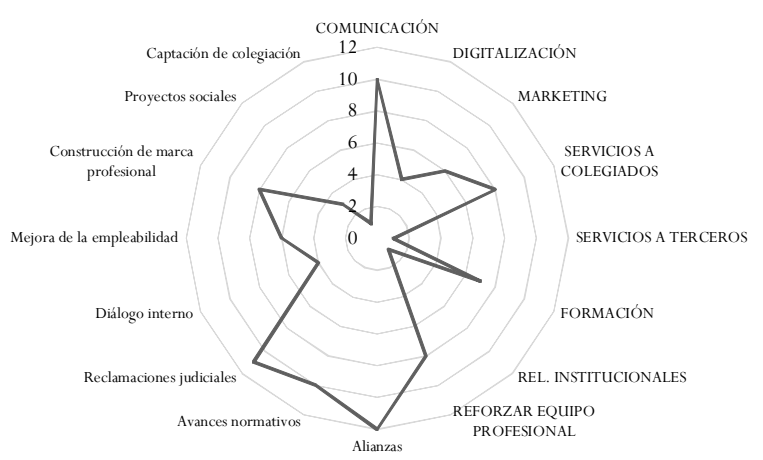

Figura 9 Esfuerzos organizativos y estratégicos que consideran desde los COLEF

\section{Discusión}

Para Botello, Casado y Germán (2015), que la mujer se haya ido incorporando a las profesiones sanitarias no tiene correspondencia con los puestos de responsabilidad, tampoco en las directivas de los colegios profesionales. Entre 251 colegios profesionales estudiados, en el $21,91 \%$ la presidencia estaba ocupada por una mujer, y el $42,80 \%$ del total de las juntas directivas. En particular, en los COLEF autonómicos la presencia de mujeres en las juntas de gobierno es actualmente del $32 \%$, y en las presidencias del 35\%.

Para que los Colegios Profesionales pudiesen adaptarse y compartir procedimientos que desde años utilizan las empresas para analizar los riesgos potenciales de una entidad, Sánchez (2016) compartió una guía que pretende trasladar la importancia de poder anticipar los asuntos que pudiesen suponer un riesgo presente y futuro, fundamentalmente basándose en un modelo de compliance (procedimientos y buenas prácticas adoptados por las organizaciones para identificar y clasificar los riesgos) y en la resiliencia de cada entidad. En concreto se enfocan tres bloques de riesgos: normativos, estratégicos, y funcionales; para a continuación realizar un planteamiento de como priorizarlos y atenderlos de la manera más eficiente posible.

El establecimiento de un modelo tendría que valorarse presupuestariamente en función del tamaño de la entidad, riegos potenciales, litigiosidad, etc. La existencia de diferentes grupos de interés y vinculados de manera interna y externa con el futuro de una organización-que influyen en sus acciones y se ven afectadas por las decisiones de esta-el foco de interés es satisfacer las necesidades de todos los agentes implicados (AECA, 2016). Es necesario tener en cuenta que la rentabilidad del control interno tiene que compararse con los beneficios esperados de la reducción de riesgos. En consecuencia, al asumir el modelo también se hace con unos costes periódicos, el más importante el de personal, consultoría, recursos materiales. Pese a este coste, un mejor control de riesgos traería aparejado una mayor capacidad de gestión de los recursos y de mejorar relaciones con los stakeholders como consecuencia de una mejor imagen corporativa entre los agentes sociales, en la línea en la que trabajan el Colegio de Abogacía de Barcelona (2021), el Colegio de Médicos de Tenerife (2021), o la Asociación Española de Administración de Empresas (2016).

Pese a que Colegios Profesionales son garantes sociales no por ello gozan de una mejor imagen social. 
Son cada vez las obligaciones y tareas a las que tienen que atender, lo que no les deja mucho margen de movimiento. Aunque ninguno de los COLEF se ha animado a ofrecer un servicio de conciliación o mediación, que sí se puede observar por ejemplo en otros ámbitos profesionales como la psicología, educación social o la abogacía, algunos se han decantado por otros servicios que consideran más útiles, como sellos de calidad, peritajes técnicos o asesoramientos a entidades. En general han realizado un intenso esfuerzo por implementar facilidades en el alta colegial, la bolsa de empleo o el campus de formación (Robles, 2014).

Cuando nos referimos a la mejor adaptación de las organizaciones hablamos normalmente de sus planes, y que éstas permitan detectar y priorizar las necesidades, ayuden a la gestión y apoyen a los órganos de gobierno. Los colegios necesitan herramientas que les doten de mayor capacidad de análisis interno y externo (Sánchez, 2015), pues pese a que no lo tienen instrumentalizado consideran que hay que poner esfuerzo prioritario en reforzar el equipo profesional, formación, alianzas, normativas, reclamaciones judiciales y marca profesional. Entre los aspectos que no son prioritarios está la organización y el diálogo interno. A este respecto, se ha reportado por parte de los COLEF la existencia de algunos planes de calidad (AGE, 2021), comunicación (Flores, 2012), o riesgos (Sánchez, 2016), pero ninguno de digitalización (GE, 2021) o carrera profesional (García, 2006). Es relevante el número de entidades colegiales que no utiliza la planificación de forma sistémica.

Todas las corporaciones participantes son conscientes de que la comunicación es cada vez más determinante. Particularmente, la comunicación interna en los colegios profesionales es singular y diferente al mundo de la empresa, pese a que hayan sido adoptados modelos «corporate» o de comunicación empresarial sin previa reflexión cualitativa (Robles, 2014). En este sentido Flores (2012), afirma en su investigación que en los colegios predomina la comunicación informal y la formal descendente, y que no poseen planes de comunicación, encontrándose en la mayoría de ocasiones con miembros pasivos. Pese a que algunos colegios profesionales puedan haber integrado satisfactoriamente esta función comunicativa, sería recomendable procurar, según la autora, una multidireccionalidad, llamando también a la iniciativa de las personas colegiadas, y de forma más cercana, pues finalmente esto influirá positivamente en la organización de la corporación.

En este caso, se hace preciso definir bien el público del colegio profesional, pues las personas colegiadas tie- nen un doble perfil, que cumple con lo característico de un trabajador, pero también de público externo. Así pues, también en los colegios profesionales, las nuevas herramientas de comunicación se conciben para un uso dual comunicativo in-out, de ahí que haya crecido el interés en su manejo y potencialidades, sobre todo de aquellas digitales. Sin embargo, aun cuando la mayoría de COLEF ha optado por externalizar los servicios de asesoría y comunicación, no consideran necesarios aquellos dirigidos a la digitalización, de ahí también el lento avance de la firma electrónica, el sistema de votación telemática, $\mathrm{u}$ otros servicios electrónicos.

Un pilar fundamental de cualquier entidad colegial son las relaciones institucionales, y aunque el vínculo con plataformas sectoriales, sindicales, políticas o públicas es escasa o nula, una gran parte de COLEF pretende seguir fortaleciendo su contacto actual con las universidades, departamentos de deportes, sanidad o educación. Un aspecto llamativo es que apenas se participa en los planes de estudio y las relaciones con colegios profesionales de otros ámbitos son bajas $(1,53: 1)$ o inexistentes, y polarizadas en determinadas profesiones sanitarias. Asimismo, en contra de las pocas jornadas divulgativas realizadas y los escasos contactos consolidados con entidades, los colegios creen que tienen que reforzar las redes profesionales, sociales e institucionales, fomentar la visibilidad de las profesiones colegiadas, así como garantizar la defensa de la profesión, las buenas prácticas y las normas deontológicas.

Por su influencia en la organización y gestión de los COLEF es unánime la necesidad de reforzar y diversificar los equipos profesionales, ya que las plantillas se nutren mayoritariamente de personas contratadas a tiempo parcial y colegiadas en la propia entidad. Aunque caracterizadas por un fuerte compromiso, un ejemplo lo encontramos durante el periodo más intenso del estado de alarma, en el que continuó el funcionamiento de los colegios con el 100\% de teletrabajo de sus plantillas, y una media aproximada del 98\% de cumplimiento (Unión Profesional, 2020), las plantillas no alcanzan para atender suficientemente la multiplicidad de tareas, cada vez más especializadas, y el ritmo de crecimiento de la colegiación en los últimos años.

En lo que respecta directamente a la gestión económica, pese a que como ya se indicó no existe apenas información pública al respecto, los COLEF no reconocen deudas bancarias contraídas, una cuestión que tiene una interpretación positiva, pues les permite manejar su liquidez de forma neutra en los próximos ejercicios, y si tuviesen alguna necesidad podrían incluso conside- 
rar previamente otros mecanismos de solidaridad corporativa. Con todo, casi un tercio admite problemas puntuales de tesorería, lo que puede guardar relación con el peso financiero de las cuotas de colegiación (Intercol.colegial, 2017), que en los COLEF autonómicos se sitúa entre el 81-100\%, mientras que en otros colegios profesionales es menor (59,7\%). Esta situación también puede ser debida a la capacidad de prestar servicios que tienen otras entidades colegiales, también de colegiación obligatoria, pero que persiguen a aquellos que no lo hacen y proponen sanciones en caso de incumplimiento, y que ven apoyados sus ingresos mediante convenios y contratos $(8,2 \%)$, subvenciones $(6 \%)$, y otras actividades $(26,1 \%)$. Tienen aquí los COLEF también una oportunidad de valorar las opciones de generar nuevos recursos diferentes a las tradicionales contribuciones colegiales, que mayoritariamente han decidido incrementar por debajo del IPC de los últimos años, provocando que el coste económico de la colegiación sea mantenga accesible.

\section{Conclusiones}

La crisis de la COVID-19, todavía vigente, está suponiendo un reto para los Educadores/as Físico Deportivos/as por los problemas derivados de las condiciones de práctica y las restricciones e incluso cierres de sus servicios e instalaciones. Con una ciudadanía muy sensible hacia su seguridad y bienestar, la pandemia ha generado mucha información y reacciones sobre más los problemas de salud que sufre la población -algunos ya conocidos pero desatendidos-, como las posibilidades que la Educación Físico Deportiva Profesional ofrece. La incertidumbre que se ha generado por la gestión de la crisis sanitaria y social ha provocado mucha tensión y problemas, tanto en las organizaciones como entre los profesionales. Éstos, a su vez, han demandado más cercanía y apoyo de sus respectivos colegios profesionales, lo que les ha obligado a redoblar esfuerzos para poder dar la mejor respuesta. En consecuencia, la percepción sobre su utilidad y su propia visibilidad se han visto beneficiadas y ayudado a que el ritmo de nuevas colegiaciones que previamente era creciente, en general, no se haya visto afectado.

Se reconoce desde los COLEF escasa utilización de herramientas de planificación, que pudiera deberse a la propia cultura organizativa, de control o de auditoría. Sería recomendable que, sin mayor dilación, se fuesen analizando las necesidades y posibilidades de cada colegio profesional para ir incorporándolas progresivamen- te a su funcionamiento.

Las entidades colegiales admiten la necesidad de conocer con más profundidad a sus colegiados e incrementar y potenciar sus estrategias de fidelización y servicios, sobre todo a través de plataformas y herramientas digitales. A pesar de las facilidades que puedan ofrecer las nuevas tecnologías, esto conlleva importantes esfuerzos en comunicación interna para ampliar tanto las vías de comunicación como las formas para lograr una mayor implicación de la colegiación en los asuntos profesionales.

Tienen los COLEF un margen amplio para mejorar las relaciones institucionales y su participación en lugares influyentes para el debate y decisión, también con colegios de otros ámbitos o entre los propios profesionales. Reconocido es que establecer relaciones permanentes y activas con diferentes entidades de referencia, sean del ámbito o no, públicas o privadas, ayudan a trasladar con mayor facilidad las propias solicitudes y realizar diálogos productivos.

Una cuestión que condiciona la gestión interna de los COLEF es su financiación, que proviene mayoritariamente de las cuotas colegiales, siendo excepcionales los ingresos por otros servicios o productos. Esta forma casi exclusiva de obtener ingresos permite un relativo confort económico a la par que un sostenido crecimiento relativo de los colegios, aunque pudieran no ser suficientes como para diversificar, ampliar y reforzar las plantillas profesionales, atender a proyectos estratégicos a medio y largo plazo, o realizar cambios organizativos que conlleven esfuerzos mayores. En la actualidad varios colegios profesionales están atendiendo servicios de consultoría y peritaje que podrían convertirse en ingresos recurrentes. Asimismo, la concurrencia con proyectos subvencionables o la elaboración de sellos de calidad del sector deportivo pudieran ser alternativas a la generación de beneficios.

Se puede mencionar que este estudio no sigue una metodología propia de los modelos de calidad total, ni utiliza herramientas como los estándares internacionales u otras herramientas industriales de gestión, lo que podría considerarse una limitación.

Entre las futuras líneas de trabajo queda abierta una profundización en el estudio de los recursos y necesidades de los Colegios Profesionales de Educación Física en España, en cuanto a su gestión, a la par que un análisis de otros modelos internacionales de organización. Igualmente, se plantea incorporar la valoración de las personas colegiadas para complementar la información obtenida desde las juntas directivas y equipos profesionales. 


\section{Referencias}

Administración General del Estado (2021): Calidad en las Administraciones Públicas. Disponible en: https:// transparencia.gob.es/transparencia/ transparencia_Home/index/PublicidadActiva/ PlanificacionYEstadistica/CalidadAAPP.html

Asociación Española de Administración de Empresas (2016): Responsabilidad Social Corporativa Interna. Disponible en: https://aeca.es/responsabilidad-social-corporativa/

Boletín Oficial Canarias (2009): Anuncio de 22 de julio de 2009, por el que se procede a la publicación de la modificación de los Estatutos del Colegio Oficial de Licenciados en Educación Física de Canarias. Disponible en: http:// www.gobiernodecanarias.org/boc/2009/154/ 022.html

Boletín Oficial Castilla y León (2018): Orden EYH/1189/ 2018, de 23 de octubre, por la que se inscribe en el Registro de Colegios Profesionales y Consejos de Colegios de Castilla y León el Estatuto particular del Colegio Oficial de Licenciados en Educación Física y en Ciencias de la Actividad Física y el Deporte de Castilla y León. Disponible en: https://bocyl.jcyl.es/html/ 2018/11/12/html/ BOCYL-D-12112018-8.do

Boletín Oficial Comunidad Madrid (2014): Resolución de 5 de noviembre de 2014, de la Dirección General de Seguridad e Interior, por la que se dispone la publicación de la modificación del Estatuto del Colegio Oficial de Licenciados en Educación Física y en Ciencias de la Actividad Física y del Deporte de la Comunidad de Madrid. Disponible en: https: / /www.bocm.es/2009-0805040820090017?ajax_popup=1

Boletín Oficial Comunidad Valenciana (2000): Resolución de 2 de febrero de 2000, de la Secretaría General de la Conselleria de Justicia y Administraciones Públicas, por la que se inscribe la adaptación a la Ley 6/1997, de 4 de diciembre, del Ilustre Colegio Oficial de licenciados en Educación Física y en Ciencias de la Actividad Física y del Deporte de la Comunidad Valenciana. Disponible en: https://n9.cl/e99xb

Boletín Oficial de Aragón (2001): Orden de 17 de agosto de 2001, del Departamento de Presidencia y Relaciones Institucionales, por la que se dispone la inscripción en el Registro de Colegios Profesionales y de Consejos de Colegios de Aragón de los Estatutos del Colegio Oficial de Licenciados en Educación Física y en Ciencias de la Actividad Física y del Deporte de Aragón. Disponible en: https://n9.cl/ 3au11

Boletín Oficial de La Rioja (2018): Resolución 112 / 2018, de la Dirección General de Justicia e Interior, por la que se declara la calificación de legalidad de carácter favorable de los estatutos del Colegio Oficial de Licenciados en Educación Física y del Deporte. Disponible en: ttps://n9.cl/ 34woy

Boletín Oficial de Navarra (2004): Resolución 717 / 2004, de 19 octubre, del Director General de Presidencia, por la que se inscribe el Colegio Oficial de Licenciados en Educación Física y Ciencias de la Actividad Física y el Deporte en el Registro de Colegios Profesionales y Consejos de Colegios de Navarra, y la de sus Estatutos en el Boletín Oficial de Navarra. Disponible en: https://www.navarra.es/ home_es/Actualidad/BON/Boletines/2004/151/ Anuncio-7/

Boletín Oficial del Estado (1974): Ley 2/1974, de 13 de febrero, sobre Colegios Profesionales. $\mathrm{N}^{\circ} 40$, de 15 de febrero de 1974. Última modificación: 19/09/2020. Referencia: BOE-A-1974-289.

Boletín Oficial del Estado (2012): Modificación de la Ley 2/1974, de 13 de febrero, de Colegios Profesionales. Disponible en: http://www.boe.es/boe/dias/2012/ 07/07/pdfs/BOE-A-2012-9112.pdf

Boletín Oficial del Estado (2013): Ley 19/2013, de 9 de diciembre, de transparencia, acceso a la información pública y buen gobierno. Disponible en: https:// www.boe.es/eli/es/l/2013/12/09/19/con

Boletín Oficial del Estado (2021): Real Decreto 472 /2021, de 29 de junio, por el que se incorpora al ordenamiento jurídico español la Directiva (UE) 2018/958, del Parlamento Europeo y del Consejo, de 28 de junio de 2018, relativa al test de proporcionalidad antes de adoptar nuevas regulaciones de profesiones. Disponible en: https:// www.boe.es/eli/es/rd/2021/06/29/472

Boletín Oficial del Principado de Asturias (2017): Resolución de 14 de marzo de 2017, de la Consejería de Hacienda y Sector Público, por la que se declara la adecuación a la legalidad de los estatutos particulares del Colegio Oficial Licenciados en Educación Física y en Ciencias de la Actividad Física y del Deporte del Principado de Asturias. Disponible en: https://n9.cl/ghq4

Boletín Oficial Islas Baleares (2014): Resolución del vicepresidente y consejero de Presidencia de 18 de agosto de 2014, en ejercicio de las competencias en materia de colegios profesionales, por la cual se califican positivamente los Estatutos del Ilustre Colegio Oficial de Licenciados en Educación Física y Ciencias de la Actividad Física y del Deporte de Baleares, dentro del marco de un procedimiento de apertura de hoja registral y se ordena su inscripción en el Registro de Colegios Profesionales de las Islas Baleares. Disponible en: https://n9.cl/rc2o4 
Boletín Oficial Junta de Andalucía (2018): Orden de 1 de octubre de 2018, por la que se aprueba la modificación de los Estatutos del Colegio Oficial de Licenciados en Educación Física y en Ciencias de la Actividad Física y del Deporte de Andalucía y se dispone su inscripción en el Registro de Colegios Profesionales de Andalucía. Disponible en: https: / /www.juntade andalucia.es/boja/2018/197/ BOJA18-197-00029-16442-01_00143718.pdf

Boletín Oficial País Vasco (1998): Orden de 24 de julio de 1998 del Consejero de Justicia, Economía, Trabajo y Seguridad Social por la que se aprueban los Estatutos del Colegio Oficial de Profesores y Licenciados en Educación Física del País Vasco. Disponible en: https:// www.euskadi.eus /y22-bopv / es / bopv2 / datos / 1998/10/9804404a.pdf

Boletín Oficial Región de Murcia (2002): Resolución de la Secretaría General de la Consejería de Presidencia de 18 de marzo de 2002 por la que se dispone la publicación de los Estatutos del Ilustres Colegio Oficial de Licenciados en Educación Física y en Ciencias de la Actividad Física y del Deporte de la Región de Murcia. Disponible en: https:/ / w w w borm. e s / bor m / d o c u men to ?obj=anu\&id $=63394$

Botello, A., Casado, R., y Germán, C. (2015): Presencia de las mujeres en los órganos de dirección de los colegios profesionales del ámbito de la salud en 2015. Rev Esp Salud Pública;89: 1-6 N. 6 - NoviembreDiciembre.

Chacón, F. (2009): La formación en los colegios profesionales, el aprendizaje a lo largo de la vida, la acreditación profesional y la calidad de servicios a los usuarios. Arbor:Ciencia, Pensamiento y Cultura. CLXXXV. Disponible en: https://doi.org/10.3989/ arbor.2009.extran1209.

Consejo COLEF - Equipo profesional (2021): Estadisticas y evolución colegial del Consejo General de la Educación Física y Deportiva. Anuario 2020. Madrid.

Consejo COLEF (2018): Memoria anual del Consejo COLEF. Disponible en: https: / / www.consejo-colef.es/post/ memoria-2018

Consejo COLEF (2019): Código de Buen Gobierno de la Organización Colegial de la Educación Física y Deportiva. Disponible en: https: / /drive.google. com/file/d/ 14TeWDoYXb7o7OqZBERUFS-ilQOWg1WFB/ view

Colegio de Médicos de Tenerife (2021): Programa de Responsabilidad Social del Colegio Oficial de Médicos de Tenerife. Disponible en: https:// medicostenerife.es/responsabilidad-social-corporativa/
Consejo de Transparencia y Buen Gobierno y Unión Profesional (2016). Guía de transparencia y acceso a la información pública dirigida a los consejos y colegios profesionales y demás corporaciones de derecho público. http: / /www.unionprofesional.com/portfolio/guia-detransparencia/

Consejo Superior de Colegios de Arquitectos de España (2019): Ejercicio de la profesión, la colegiación en España. Disponible en: https: / /www.cscae.com/index.php/ 2015-01-28-11-29-51

Consejo Superior de Deportes (2004): Manual de buen gobierno en asociaciones y federaciones deportivas del CSD. Disponible en: https: / /www.csd.gob.es/es/federaciones-y-asociaciones/ federaciones-deportivasespanolas/codigo-de-buen-gobierno

Contreras, J.J. \& Crespo, M.F. (2005): ¿Hacia un nuevo sentido de la gerencia? Revista Venezolana de Gerencia, vol. 10, núm. 32, octubre-diciembre, pp. 583-604. Universidad del Zulia. Maracaibo, Venezuela.

Diario Oficial Castilla-La Mancha (2013): Resolución de 10/06/2013, de la Viceconsejería de Presidencia y Administraciones Públicas, por la que se publican los Estatutos del Colegio Oficial de Licenciados en Educación Física y en Ciencias de la Actividad Física y del Deporte de Castilla-La Mancha. Disponible en: http:// docm.castillalamancha.es / portaldocm / $\mathrm{d}$ e $\mathrm{t}$ a $\mathrm{l}$ l e $\mathrm{D}$ o $\mathrm{c}$ u $\mathrm{m}$ e $\mathrm{n}$ t o. do?idDisposicion $=1371559112252870580$

Diario Oficial de Extremadura (2005): Resolución de 16 de junio de 2005, de la Consejera de Presidencia, por la que se acuerda la publicación de la adaptación de los Estatutos del Colegio Oficial de Licenciados en Educación Física y en Ciencias de la Actividad Física y el Deporte de Extremadura a la Ley 11 /2002, de 12 de diciembre, de Colegios y de Consejos de Colegios Profesionales de Extremadura. Disponible en: http://doe.gobex.es/pdfs/doe/2005/ $790^{\circ} / 05061669$.pdf

Diario Oficial de Galicia (2020): Decreto 107/2020, de 23 de julio, por el que se aprueban los estatutos del Colegio Oficial de Licenciados en Educación Física y en Ciencias de la Actividad Física y del Deporte de Galicia. Disponible en: https: / /www.xunta.gal/dog/Publicados/2020/ 20200810/AnuncioG0244-280720-0003_gl.html

Diario Oficial Generalitat de Catalunya (2000): Decret 302/2000, de 31 d'agost, de canvi de denominació del Col-legi de Professors i Llicenciats d'Educació Física de Catalunya. y Resolución JUS/2679/2013, de 29 de noviembre, por la que se inscriben en el Registro de Colegios Profesionales de la Generalidad de Cataluña los Estatutos del Colegio de Licenciados en Educación Física y Ciencias 
de la Actividad Física y del Deporte de Cataluña. Disponible en: https://n9.cl/n6h8j

Díez, C. \& Gambau, V. (2019): Perspectiva histórica del educador físico. Revista Española de Educación Física y Deportes. Número 422, año LXX, 3er trimestre.

Elías, J. \& Mascaraya, J. (1998): Más allá de la comunicación interna: La intracomunicación; $2^{a}$ Edición. Edit. Gestión 2000.

Flores, I. (2012): La gestión de la comunicación en los colegios profesionales. Anuario Electrónico de Estudios en Comunicación Social «Disertaciones», 5 (2), Artículo 9. Disponible en: http://erevistas.saber.ula.ve/ index.php/Disertaciones/

Gambau, V. (2018): Historia de la profesión de los titulados universitarios en ciencias de la actividad física y del deporte. Disponible en: https: / /www.consejo-colef.es/ post/vgambau-historia-profesion

García, J. (2006): Modelos de incentivación y carrera profesional. RevistaAEN. Vol. XXVI, n97, enero/marzO: 89-104.

García, M. \& Pradas, M. (2016): ¿La transparencia de las páginas web ayudan en la captación de clientes? Análisis de los campos de golf en Andalucía. Intangible Capital. 12(3): 805-821. Disponible en: https://n9.cl/2f808

Gobierno de España (2021): Plan de digitalización de las administraciones públicas y estrategias TIC. Portal Administración Electrónica. Disponible en: https:// administracionelectronica.gob. es/pae_Home/ pae_Estrategias / Estrategia-TIC/PlanDigitalizacion-AAPP.html\#.YJUm3qHtaUk

Il-lustre Col·legi de l'Advocacia de Barcelona (2021): SolidarICAB - Responsabilidad Social Corporativa. Disponible en: https: / / www.icab.es/es/colegio/conoce-el-colegio/responsabilidad-social-corporativa/

Instituto Nacional de Estadística (2019): Estadísticas de profesionales colegiados 2018. Disponible en: https: / /www.ine.es.

Intercol.legial (2017): Memoria de actividades 2017. Disponible en: https://n9.cl/izrk

Múzquiz, G. (2012): ¿Qué más pueden hacerlas organizaciones profesionales en materia de empleo, empleabilidad e internacionalización?. Estudios UP. Disponible en: http:/ /www. unionprofesional.com/portfolio/estudio-empleo-empleabilidad/internaciónalizacion/

Múzquiz, G. (2013): Desarrollo Profesional Continuo: una herramienta para la movilidad y la internacionalización. Serie Estudios Unión Profesional. Disponible en: http: / / www.unionprofesional.com/portfolio/desarrollo-profesional-continuo/

Palomar Olmeda, A. (2019). Viabilidad de una ley estatal ordenadora de la profesión. Revista Española de Educación Física y Deportes, 425, 103-130.

Robles Gómez, M.A. (2014). La comunicación interna en los colegios profesionales: el caso de la farmacia andaluza. (Tesis Doctoral Inédita). Universidad de Sevilla, Sevilla. Disponible en: https://hdl.handle. net / $11441 / 78484$

Sánchez, E. (2015): Valoración de entidades colegiales. Estudios Unión Profesional. Disponible en: http:// www.unionprofesional.com / portfolio/ valoraciondeentidades-colegiales /

Sánchez, E. (2016): Análisis global de riesgos en las organizaciones colegiales. Marco para realizar un modelo de prevención de riesgos y cumplimiento normativo en las corporaciones colegiales. Serie Estudios Unión Profesional. Disponible en:http: / / www.unionprofesional.com/ prevencion-de-riesgos-y-cumplimiento-normativoen-las-corporaciones-colegiales /

Unión Profesional (2013): El Ejercicio de las Profesiones Tituladas en el Marco de los Derechos Fundamentales. Disponible en: http://www.unionprofesional.com/ portfolio/estudio-1/

Unión Profesional (2016): ¿Qué son y para qué sirven los colegios profesionales?. Consejo General del Trabajo Social. Disponible en: http:// www.unionprofesional.com/que-son-los-colegiosprofesionales-y-para-que-sirven/

Unión Profesional (2020). Las profesiones colegiadas ante la covid-19. Serie Estudios de Unión Profesional. Madrid. Disponible en: http:// www.unionprofesional.com/portfolio/guia-las-profesiones-colegiadas-ante-el-covid-19/

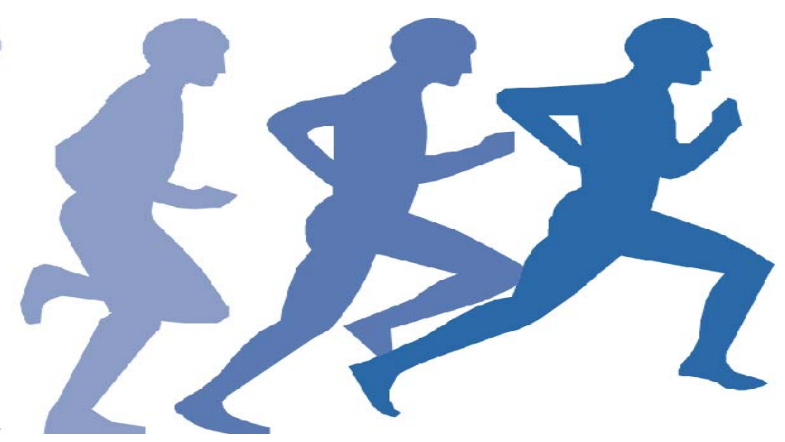

